\title{
GENERATING GROUPS FOR NILPOTENT VARIETIES
}

\author{
FRANK LEVIN 1 \\ (Received 2 May 1968; revised 14 October 1968)

\begin{abstract}
To Bernhard Hermann Neumann on his 60th birthday
Communicated by G. E. Wall
\end{abstract}

Let $\mathfrak{N}_{c}$ denote the variety of all nilpotent groups of class $\leqq c$, that is, $\mathfrak{N}_{c}$ is the class of all groups satisfying the law

$$
\left[x_{1}, \cdots, x_{c+1}\right]=1 \text {, }
$$

where we define, as usual, $\left[x_{1}, x_{2}\right]=x_{1}^{-1} x_{2}^{-1} x_{1} x_{2}$ and, inductively, $\left[x_{1}, \cdots, x_{n}\right]=\left[\left[x_{1}, \cdots, x_{n-1}\right], x_{n}\right]$. Further, let $F_{k}\left(\mathfrak{N}_{c}\right)$ denote a free group of $\mathfrak{R}_{c}$ of rank $k$. In her book Hanna Neumann ([4], Problem 14) poses the following problem: Determine $d(c)$, the least $k$ such that $F_{k}\left(\mathfrak{R}_{c}\right)$ generates $\mathfrak{N}_{c}$. Further, she suggests, incorrectly, that $d(c)=[c / 2]+1$. However, as we shall prove here, the correct answer is $d(c)=c-1$, for $c \geqq 3 .{ }^{2}$ More generally, we shall prove the following result.

TheOREM. Let var $F_{i}\left(\mathfrak{R}_{c}\right)$ denote the variety generated by $F_{i}\left(\mathfrak{R}_{c}\right)$. Then

$$
\operatorname{var} F_{1}\left(\mathfrak{R}_{c}\right)<\operatorname{var} F_{2}\left(\mathfrak{R}_{c}\right)<\cdots<\operatorname{var} F_{c-1}\left(\mathfrak{R}_{c}\right)=\mathfrak{R}_{c} \quad \text { for all } c \geqq 3 \text {. }
$$

For convenience we will divide the proof into two parts. In part I the inequalities in (1) are established by constructing, for each $k \leqq c$, a law in $F_{k-2}\left(\Re_{c}\right)$ which is not a law in $F_{k-1}\left(\mathfrak{R}_{c}\right)$. In part II the final equality in (1) is established by showing that $F_{k}\left(\mathfrak{R}_{c}\right)$ is residually a $(c-1)$ generator group, for any $k \geqq c .^{3}$

\section{Part I:}

$$
\operatorname{var} F_{k-1}\left(\Re_{c}\right)<\operatorname{var} F_{k}\left(\Re_{c}\right), \quad 2 \leqq k \leqq c-1 .
$$

Proof of PART I. To show (2) for $3 \leqq k \leqq c-1$ (the case $k=2$ is trivial) it is sufficient to find a law $Q_{k, c}=1$ which holds in $F_{k-1}\left(\mathfrak{R}_{c}\right)$ but

1 The autbor gratefully acknowledges the support of the National Science Foundation.

2 Since preparing this manuscript the author has received a letter reporting two independent proofs of this result from M. F. Newman in Canberra [1], both based on somewhat less elementary arguments, however.

3 All notation and terminology not specified follows that of [3] or [4]. 
not in $F_{k}\left(\mathfrak{R}_{c}\right)$. The particular law we have chosen is constructed as follows: Let

$$
Q_{k}=\prod_{\sigma}\left[x_{k}, x_{\sigma(1)}, \cdots, x_{\sigma(k-1)}\right]^{|\sigma|}
$$

where $\sigma$ runs through all permutations of $\{1, \cdots, k-1\}$ and $|\sigma|=1$ if $\sigma$ is even, $|\sigma|=-1$ if $\sigma$ is odd. Then we define $Q_{e-1, c}$ to be $Q_{c}$ and, for $3 \leqq k \leqq c-2, Q_{k, c}=\left[Q_{k+1}, x_{k+2}, \cdots, x_{c}\right]$. We first prove the following.

(A) $Q_{e}=1$ holds in $F_{e-2}\left(\mathfrak{N}_{c}\right)$ but not in $F_{c-1}\left(\mathfrak{N}_{c}\right), c \geqq 3$.

PROOF of (A). Let $R_{k}=Z\left[y_{1}, \cdots, y_{k}\right]$ be a free associative ring over $Z$ in the free non-commuting indeterminates $y_{1}, \cdots, y_{k}$, and let $I_{k, c+1}$ be the (two-sided) ideal in $R_{k}$ generated by all monomials of degree $c+1$. In $R_{k, c}=R_{k} / I_{k, c+1}$ any element $1+y_{i}$ has an inverse $1-y_{i}+y_{i}^{2}-\cdots \pm y_{i}^{c}$, and, hence, we may consider the multiplicative group $G_{k, c}$ in $R_{k, c}$ generated by the elements $1+y_{i}, i=1, \cdots, k$. We define $\left(z_{1}, z_{2}\right)=z_{1} z_{2}-z_{2} z_{1}$ and, inductively, $\left(z_{1}, \cdots, z_{n}\right)=\left(\left(z_{1}, \cdots, z_{n-1}\right), z_{n}\right), z_{i} \in R_{k}$. A direct computation shows that

$$
\left[1-z_{1}, \cdots, 1+z_{n}\right]=1+\left(z_{1}, \cdots, z_{n}\right)+\text { terms of higher degree, }
$$

for any $1+z_{i} \in G_{k, c}$. Since the $n$-length commutator $\left(z_{1}, \cdots, z_{n}\right)$ is a homogeneous polynomial of degree $n$ in the $z_{i}$, it follows that $G_{k, c} \in \mathfrak{N}_{c}$. In fact (cf., [3], Chapter 5), $G_{k, c} \cong F_{k}\left(\mathfrak{N}_{c}\right)$. In particular,

$$
\prod_{\sigma}\left[1+z_{\mathfrak{c}}, 1+z_{\sigma(1)}, \cdots, 1+z_{\sigma(c-1)}\right]^{|\sigma|}=1+\sum_{\sigma}|\sigma|\left(z_{c}, z_{\sigma(1)}, \cdots, z_{\sigma(c-1)}\right),
$$

for elements $1+z_{i} \in G_{k, c}$. Hence, to prove (A) it is sufficient to prove

$$
Q_{c}^{\prime}=\sum_{\sigma}|\sigma|\left(x_{c}, x_{\sigma(1)}, \cdots, x_{\sigma(c-1)}\right)=0
$$

in $R_{c-2, c}$ but not in $R_{c-1, c}$. (In this context, $Q_{c}^{\prime}$ may be considered as element in the free associative ring $R_{c}(x)=Z\left[x_{1}, \cdots, x_{c}\right]$ just as $Q_{c}$ may be considered as an element of the free group on $x_{1}, \cdots, x_{c}$ (cf., [3]).)

The proof of $(5)$ is based on the following lemma.

Lemma. Let $P \neq 0$ be a homogeneous polynomial in $R_{c}$ of total degree $c(\geqq 3)$ and of degree 1 in each indeterminate $y_{1}, \cdots, y_{c}$. If $P$ is a linear combination of c-fold commutators, i.e., elements of the form $\left(a_{1}, \cdots, a_{c}\right)$, then for some $i \neq j, P \neq 0$ modulo $y_{i}=y_{j}$. (The latter statement will be abbreviated by $P\left\{y_{i}=y_{j}\right\} \neq 0$.)

Proof. First we note that the polynomial

$$
P_{c}=P_{c}\left(y_{1}, \cdots, y_{c}\right)=\sum_{\sigma}|\sigma| y_{\sigma(1)} \cdots y_{\sigma(c)}
$$


where $\sigma$ runs through all permutations of $\{1, \cdots, c\}$ satisfies $P_{b}\left\{y_{i}=y_{i}\right\}=0$, for any $i \neq j . P_{n}, c \geq \mathbf{3}$. is not a linear combination of $c$-fold commutators. For, if it were, then by the Dynkin-Soecht-Wever Theorem (cf., [3]), we would have

$$
\left\{P_{c}\right\}=\sum_{\sigma}|\sigma|\left(y_{\pi(1)}, \cdots, y_{\pi(r)}\right)=c P_{c} .
$$

However, a straightforward induction starting with

$$
P_{3}=2\left(x_{1}, x_{2}, x_{3}\right)+2\left(x_{2}, x_{3}, x_{1}\right)+2\left(x_{3}, x_{1}, x_{2}\right),
$$

which is 0 by the Tacobi identitv. and noting that, for $c>\mathbf{3}$,

$$
\left\{P_{c}\right\}=\sum_{k=1}^{\infty} \sum_{\pi(c)=k_{i}}|\sigma|\left(y_{\sigma(1)}, \cdots, y_{\sigma(c)}\right),
$$

shows that $\left\{P_{c}\right\}=0$ for all $c \geq \mathbf{3}$.

To complete the proof of the Lemma it suffices to show that any $P$ described in the Jemma. which satisfies $P\left\{y_{i}=y_{i}\right\}=0$ for all $i \neq i$, is a multiple of $P_{c}$. The proof is by induction on $c$ (starting with $c=2$. however). For $c=2, P=n y_{1} y_{2}+m y_{2} y_{1}$, and $P\left\{y_{1}=y_{0}\right\}=0$ implies that $m==-n$, j.e.. $P=n P_{2}$.

Next. let $c>2$ and write $P$ in the form

$$
P=\sum_{k=1}^{\infty} A_{k} y_{k}
$$

where the $A_{\text {; }}$ are homogeneous of total degree $c-1$ in $y_{1}, \cdots, \hat{y}_{1}, \cdots, y_{c}$ $\left(y_{i}\right.$ omitted). Since $P\left\{y_{v}=y_{a}\right\}=0$ for any $p, q \neq k$. it follows by induction that

$$
A_{k}=n_{k} P_{n-1 . k},
$$$$
n_{k} \in Z \text {, }
$$

where $P_{c-1, k}=P_{c-1}\left(y_{1}, \cdots, \hat{y}_{k}, \cdots, y_{c}\right)$, as defined by (6). Thus,

$$
P=\sum_{k=1}^{c} n_{k} P_{c-1 . k} . y_{k},
$$

However, since $P\left\{y_{1}=y_{k}\right\}=0$ for any $k \neq 1$, it follows from a, comparison of the first and $k$-th summands in (7) that this is possible only if

$$
n_{1} P_{n-1.1} y_{1}+n_{k} P_{c-1 . k} y_{k}=n_{1} \sum_{\sigma}|\sigma| y_{\sigma(1)} \cdots y_{r(n)},
$$

where the summation is restricted to all those $\sigma$ for which either $\sigma(c)=1$ or $\sigma(c)=k$. Since this is to be true for all $k$. it follows that $P=n_{1} P_{n}$. This proves the I.emma.

We may now apply the Lemma to (5). Since the component of $Q_{c}^{\prime}$ (considered as a polynomial in $R_{r}$ ) of terms with left factor $x_{r}$ is precisely 


$$
\sum_{\sigma} x_{c} x_{\sigma(1)} \cdots x_{\pi(c-1)},
$$

it is clear that $Q_{n}^{\prime} \neq 0$. Further, $Q_{n}^{\prime}$ is antisvmmetric in the $x_{1}, \cdots, x_{n \rightarrow 1}$, so that $Q_{,}^{\prime}\left\{x_{i}=x_{i}\right\}=0$ for any $i, j \neq c, i \neq i$. Since $Q_{c}^{\prime} \neq 0$. it follows from the Lemma. that $Q_{s}^{\prime}\left\{x_{s}=x_{i}\right\} \neq 0$ for some $i \neq c$. Thus. $Q_{a}^{\prime}=0$ is not a law in $R_{n-1 . n}$, which means that $Q_{n}=1$ is not a. law in $F_{n-1}\left(\Re_{n}\right)$ as well.

As just observed. $Q_{0}^{\prime}=0$ if any two of the $x_{x}, \cdots, x_{r \ldots 1}$, are identified. Thus, in $R_{c-9 .}$ if the $x_{i}$ are replaced by the $y_{i}$, then since there are just. $c-2$ distinct $y_{i}$ it follows that $Q_{c}^{\prime}$ will vanish. To decide whether $Q_{n}^{\prime}=Q_{n}^{\prime}\left(x_{1}, \cdots, x_{n}\right)$ (i.e., considered as a. function of the $x_{i}$ ) vaniches over all of $R_{n_{\ldots, a}, \text { or not }}$ it is enough modulo $I_{n-2, n+1}$ to consider linear substitutions of the $y_{i}$ for the $x_{i}$. However. since $Q_{n}^{\prime}\left(x_{1}, \cdots, x_{n}\right)$ is multilinear in the $x_{i}$. such a, substitution yields a linear combination of terms of the form $Q_{n}^{\prime}\left(v_{1}, \cdots, v_{c}\right)$, $v_{i} \in\left\{y_{1}, \cdots, y_{n \rightarrow-2}\right\}$. By the previous remark, each of these terms vanishes. Hence, $Q_{n}^{\prime}=0$ is a law in $R_{r-2}$ and $Q_{n}=1$ is a law in $F_{n \ldots,}\left(\Re_{r}\right)$. This completes the proof of (5) and. hence, of (A).

The above argument shows that var $F_{n-\infty}\left(\Re_{n}\right)<\operatorname{var} F_{n-1}\left(\mathfrak{N}_{c}\right)$. To complete the proof of Part I we must show that $O_{k n}$ is trivial over $F_{k-1}\left(\mathfrak{R}_{n}\right)$ but not over $F_{k}\left(\mathfrak{R}_{n}\right), 3 \leqq k \leqq c-2.4$ That $Q_{k_{-c}}=1$ is a. law for $F_{k_{-1}}\left(\mathfrak{R}_{c}\right)$ follows immediately from the above arguments regarding $Q_{n}$. Further, as we have seen above, $Q^{\prime}\left\{x_{i k}=x_{i}\right\} \neq 0$ for some $i \neq k$. This imolies, however, that

$$
\left[Q_{k}\left\{x_{k}=x_{c}\right\}, z_{k+1}, \cdots, z_{c}\right] \neq 1
$$

where, $z_{i} \in\left\{x_{1}, \cdots, x_{k}\right\}$, so that $Q_{k_{n}, \text { will }}$ not be a law for $F_{n_{k}}\left(\mathfrak{R}_{n}\right)$. This is a. direct consequence of the more general remark that if $P \in R_{n}$ is a polynomial with a.t least two distinct $u_{\text {; }}$ a.opearing in each term. then for any $y_{i},\left(P, y_{i}\right) \neq 0$. To see this. express $P$ in the form $P=\sum y_{i}^{m} A_{m}$, where the $A_{m}$ are polynomials without terms with left $u_{i}$ factors. We may assume that no $A_{m}$ has a term of degree 0 . Then.

$$
y_{i} P=\sum y_{i}^{m+1} A_{m} \neq P y_{i}=\sum y_{i}^{m} A_{m} y_{i} .
$$

This completes the proof of Part I.

\section{Part II:}

For any $k \geqq c \geqq 3, F_{k}\left(\mathfrak{N}_{e}\right)$ is residually a $(c-1)$ generator group.

ProOF of ParT II.. The proof is by induction on $c \geqq 3$. First. it is known, for any $c \geqq 3$, that the variety of metabelian groups nilpotent of

- This portion of the proof of Part 1 based on the law $Q_{k, c}$ for $k \leqq c-2$. bas been suggested to the author by M. F. Newman and is included here with bis permission. It replaces the author's original proof which was based on a slightly more comolicated law with a resulting lengthier argument. 
class $c$ is generated by its 2-generator groups (Baumslag, Neumann, Neumann, Neumann [2], cf., [4], 36. 34). This proves the case $c=3$. Next, let $c>3$ and set $F=F_{k}\left(\mathfrak{R}_{c}\right)$, for an arbitrary $k \geqq c$. By induction,

$$
F / \gamma_{c} F=F_{k}\left(\Re_{c-1}\right) \text {, }
$$

where $\gamma_{c} F$ denotes the $c$-th term of the lower central series of $F$, is residually $(c-2)$ generator. Hence, consider $g \in \gamma_{c} F$. Note that $g$ is a product of commutators of the form $\left[a_{1}, \cdots, a_{c}\right]$. If $g$ involves more than $c$ generators, say $g_{1}, \cdots, g_{m}$, set $g^{\prime}=g\left\{g_{c+1}=\cdots=g_{m}=1\right\}$. We may assume, after a possible reordering of the indices of the $g_{i}$, that $g^{\prime} \neq 1$. From the Lemma it follows that $g^{\prime}\left\{g_{i}=g_{j}\right\} \neq 1$ for some $i \neq j, i, j \leqq c$. Thus, if $N$ is the normal closure of the elements $g_{i} g_{j}^{-1}, g_{c+1}, \cdots, g_{m}$ in $F$, it follows that $g^{\prime} \notin N$, and since $F / N$ is generated by $(c-1)$ elements, it follows that $F_{k}\left(\mathfrak{R}_{c}\right)$ is residually a $(c-1)$ generator group, as desired. This completes the proof of Part II and, hence, of the theorem.

\section{References}

[1] L. G. Kovács, M. F. Newman and P. F. Pentony, 'Generating groups of Nilpotent varieties', Bull. AMS, 74 (1968), 968-971.

[2] G. Baumslag, B. H. Neumann, Hanna Neumann, Peter M. Neumann, 'On varieties generated by a finitely generated group', Math. A. 86 (1964), 93-122.

[3] W. Magnus, A. Karrass, D. Solitar, Combinatorial Group Theory (New York and London, Interscience, 1966).

[4] Hanna Neumann, Varieties of Groups (Berlin, Heidelberg and New York, Springer-Verlag, 1967).

Rutgers, The State University

New Brunswick, N.J. 08903

U.S.A. 